\title{
Antigenicity and Immunogenicity Evaluations of Plant-based VP2 Protein of Malaysian Highly Virulent Infectious Bursal Disease Virus (hvIBDV) Expressed in Nicotiana tabacum
}

\author{
Siti Hasmah Mohtar ${ }^{1,2}$, Hwei-San Loh ${ }^{1}$, Festo Massawe ${ }^{1}$, Abdul Rahman Omar ${ }^{3}$ \\ ${ }^{I}$ (School of Biosciences, Faculty of Science, University of Nottingham Malaysia Campus, Malaysia) \\ ${ }^{2}$ (Faculty of Science and Biotechnology, Universiti Selangor, Malaysia) \\ ${ }^{3}$ (Institute of Bioscience, Universiti Putra Malaysia, Malaysia)
}

\begin{abstract}
The VP2 protein of highly virulent infectious bursal disease virus (hvIBDV) carries major neutralizing epitopes which is able to induce host neutralizing antibodies. This feature makes VP2 as a suitable target protein for the development of subunit vaccine. Plant viral-based expression system has been identified as a potential system that is capable to express foreign proteins in plants. The present study was aimed at developing a vaccine candidate for the hvIBDV by constructing a chimeric plant virus of Potato virus $X(P V X)$ that capable of expressing the VP2 gene of hvIBDV in Nicotiana tabacum. Western blot analyses indicated the $V P 2$ protein was expressed either as a $50 \mathrm{kDa}$ protein or fused with the PVX coat protein of $80 \mathrm{kDa}$. Dot blot analysis of rat serum immunized with the VP2 protein from total soluble protein showed positive signal when immunoreacted against the hVIBDV antigen. Immunization of the expressed VP2 protein in specific-pathogenfree $(S P F)$ chickens revealed detection of anti-VP2 analyzed by enzyme-linked immunosorbent assay (ELISA).
\end{abstract}

Keywords - highly virulent infectious bursal disease virus, VP2 protein, Potato virus X, Nicotiana tabacum, neutralizing antibodies

\section{INTRODUCTION}

The infectious bursal disease virus (IBDV) is the causative agent of highly contagious disease in young chickens. The infections lead to high rate of morbidity and mortality in an acute condition [1]. The virus destroys the immature B lymphocytes within the bursa of Fabricus that lead to immunosuppression and subsequently increase in susceptibility with infections to other pathogens [2]. In 1987, a highly virulent strain of IBDV (hvIBDV) appeared and spread in Europe causing severe economic losses. The new strain was more virulent and penetrated through maternal antibodies that had provided protection in the past [3]. The hvIBDV infection causes economic losses to the poultry industry in Malaysia and worldwide.

Control of IBD is primarily achieved by maternal-derived antibodies induced by live and inactivated vaccines given to breeder hens and by vaccinating chicken with a live-attenuated strain of IBDV. Immunization of chicken with a live-attenuated vaccine was not providing fully protection against the infection [4]. Some chickens immunized with live attenuated vaccines show certain degree of bursal atrophy and are not fully protected against IBDV infection [5] [4]. In addition, live attenuated IBD vaccine after passage in chickens has been noted to increase in virulence [6]. The search for new approach to improve the current vaccines or to produce new vaccines is warranted in order to overcome problems associated with the currently available live attenuated vaccines against IBDV infection.

VP2 has been identified as the main host protective antigen of IBDV and carries major neutralizing epitopes [7] [8]. The VP2 protein has been expressed for the development of subunit vaccines using a variety of different expression systems including Escherichia coli [9], fowl poxvirus [10] [11], fowl adenovirus [12], herpesvirus of turkey [13], Marek's disease virus [14], baculovirus [15] [16] and yeast Pichia pastoris [17]. Since the neutralizing domains are highly conformational [7], successful vaccine development requires systems where the recombinant products mimic the authentic proteins and their conformation.

Plants have a great potential as an expression system for the production of beneficial foreign proteins such as vaccine antigen. The expression of heterologous proteins in plants has become an alternative to more current expression systems based on animal, yeast or bacteria cell culture [18] [19]. Various plant expression systems have been established for molecular farming [20]. A number of proteins of pharmaceutical and industrial applications have been produced in plants, where the low cost of production, easy scale-up and low risk of contamination with animal pathogens are the main advantages [18] [19] [21]. Additionally, plants expressing a recombinant antigen can be used directly as an oral vaccine, as long as the antigen is expressed in the palatable part of the plant. 
Foreign genes can be produced in plants by permanent insertion or by transient expression using plant virus-based vectors [22]. The transient strategy using plant virus-based vector allows high level of protein accumulation in the host plants facilitated by rapid amplification of the targeted gene during replication of infectious plant viruses [18]. Model plants such as Arabidopsis thaliana and tobacco are strong candidates for initial studies to generate stable transgenic plants expressing proteins of interest. Previous study demonstrated the successful expression of VP2 protein of IBDV in Arabidopsis thaliana by transfecting the gene via the Agrobacterium tumefaciens [23]. The protein was found to be immunogenic and protective against IBDV infection by oral and subcutaneous delivery to the chicken. Recently, a truncated VP2 protein was transiently expressed as a fusion protein with the N-terminus of Bamboo mosaic virus coat protein in Nicotiana benthamiana [24]. The purified virion containing the expressed VP2 protein immunized in chickens was found immunogenic and provide protection against the very virulent IBDV.

Previous studies on the development of a plant based vaccine focused on stable expression strategy whereby the procedure involves integration of foreign gene into the nuclear genome of the plant cells. Eventually, the strategy demonstrates drawbacks include longer time required for analysis of the transformants, unpredictable of protein expression and problem in generating sufficient transgenic plant material for protein analysis. Therefore, the present study was aimed to produce a plant based vaccine candidate for the Malaysian hvIBDV using plant viral based transient expression strategy. In the present study, a subunit vaccine candidate based on the VP2 protein of the Malaysian hvIBDV was developed by employing a plant virus-based vector that is capable in expressing the immunogenic VP2 protein of hvIBDV in Nicotiana tabacum. The complete genome of VP2 $(1.4 \mathrm{~kb})$ was cloned and expressed in a potato virus X (PVX) expression vector. The antigenicity and immunogenicity properties of the expressed VP2 protein were evaluated.

\subsection{Isolation and amplification of hvIBDV VP2 gene}

\section{MATERIALS AND METHOD}

The Malaysian strain of hvIBDV was extracted from chorioallantoic membrane (CAM), kindly provided by Prof. Dr. Abdul Rahman Omar, Institute of Bioscience, Universiti Putra Malaysia. The total RNA was extracted using TRIZOL ${ }^{\circledR}$ LS Reagent (Invitrogen, USA) according to the method described previously [25]. The RT-PCR condition was optimized to synthesize first-strand cDNA from total RNA of hvIBDV using SuperScript III First-Strand Synthesis System (Invitrogen, USA). About 50 to $100 \mathrm{ng}$ of the synthesized cDNA were used in PCR amplification of the VP2 gene using a set of primers designated as VP2F1 (5'GGGATATCATGACAAACCTGCAAGATCAAACCCAAC-3') and VP2R1 (5'-GGGTCGACTTAGGC CCGGATTATGTCTTTG-3'). The 5' end of both forward and reverse primers was incorporated with EcoRV and SalI sites, respectively.

\subsection{Cloning of VP2 gene onto Potato Virus X (PVX) virus-based vector}

The amplified product of VP2 gene was purified using MEGAquick-spin PCR and Agarose Gel DNA Extraction System (Intron Biotechnology, Korea) following the protocol recommended by the manufacturer. The Potato virus X (PVX) vector designated as pP2C2S [26] used in this study was kindly provided by Prof. Dr. David Baulcombe from the University of Cambridge, UK. The pP2C2S vector was propagated and extracted using GeneAll® Exprep ${ }^{\mathrm{TM}}$ Plasmid Quick (GeneAll Biotechnology, Korea) according to the protocol described by the manufacturer. The purified VP2 gene together with the pP2C2S vector was digested with EcoRV and SalI enzymes. The position and sequence of both EcoRV and SalI recognition sites on the pP2C2S vector were described in Fig. 1. The restriction digestion reactions consist of $1 \mu \mathrm{g}$ of recombinant plasmids, $1 \mathrm{X}$ restriction endonuclease buffer, $10 \mathrm{U}$ restriction enzymes (EcoRV and SalI) and deionized distilled water filled up to a final volume of $15 \mu \mathrm{l}$. The ligation reaction mixture was prepared in $40 \mu \mathrm{l}$ reaction volume which consisted of 10X ligation buffer, 2 U of T4 DNA ligase (New England Biolabs, UK), 100 ng PVX vector, 500 ng VP2 gene variants and filled up with distilled water. After incubated at $25^{\circ} \mathrm{C}$ for 2 hours, $4 \mu$ l of ligation reaction was transferred into a vial of XL1-Blue competent cells. The transformed bacteria were spread on to the LB agar plates containing $50 \mu \mathrm{g} / \mathrm{ml}$ ampicillin and incubated at $37^{\circ} \mathrm{C}$ for 16 hours. PCR screening of the recombinant PVX::VP2 vector was carried out using VP2F1 and VP2R1 primers as described previously. The presence of VP2 gene in the PVX vector was further verified by restriction enzyme analysis of the recombinant vectors using EcoRV and SalI enzymes. 


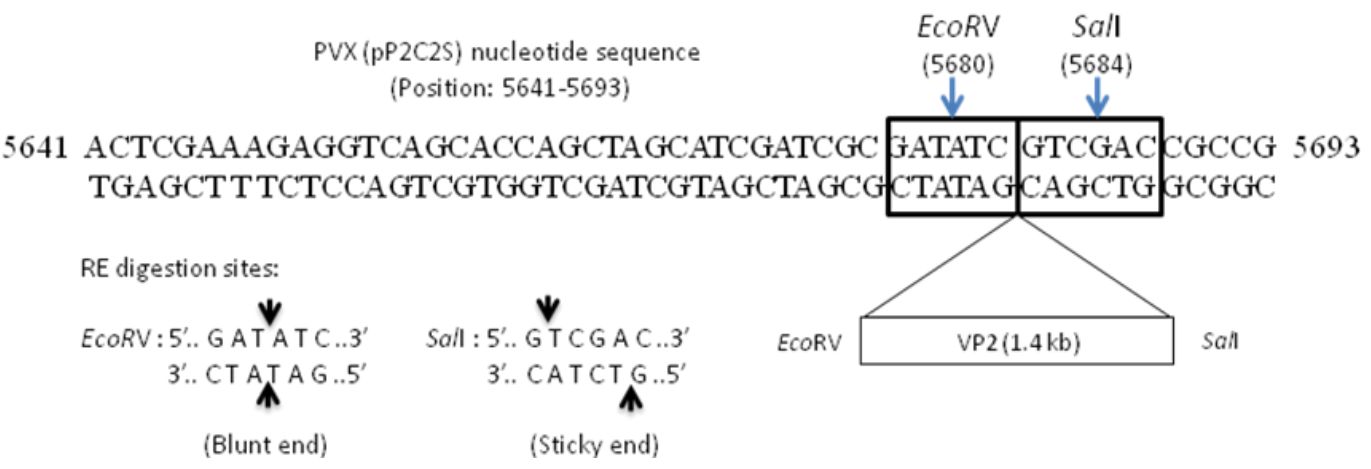

Fig. 1. Schematic diagram of nucleotide sequence of PVX (pP2C2S) vector displaying the recognition sites of EcoRV (position 5677 to 5682) and SalI (position 5683 to 5688) for the cloning of VP2 gene variants in PVX vector.

\subsection{In vitro synthesis of capped viral transcripts}

The empty PVX and the recombinant PVX::VP2 vector were linearized by single digestion with the $S p h \mathrm{I}$ restriction enzyme. The digestion reaction was terminated by adding $0.75 \mu \mathrm{l}$ of $0.5 \mathrm{M}$ EDTA, $1.5 \mu \mathrm{l}$ of $5 \mathrm{M}$ $\mathrm{NH} 4$ acetate and $30 \mu \mathrm{l}$ of ethanol and the mixture was chilled at $-20^{\circ} \mathrm{C}$ for 15 minutes. After centrifugation, the DNA pellet was resuspend in distilled water at a concentration of 0.5 to $1.0 \mu \mathrm{g} / \mu \mathrm{l}$ and subsequently used for in vitro transcription. The viral transcripts of empty PVX and recombinant PVX::VP2 were generated using Ambion mMESSAGE mMACHINE® Kits (USA) following the protocol described by the manufacturer. Capped transcription assembly reactions consisted of nuclease free water, $1 \mathrm{X}$ reaction buffer, $1 \mathrm{X}$ NTP/CAP, 2 $\mu \mathrm{l}$ of enzyme mix and $1.0 \mu \mathrm{g}$ of linearized template DNA and made up to $20 \mu \mathrm{l}$ with nuclease free water. The reaction mixture was incubated at $37^{\circ} \mathrm{C}$ for 15 minutes for cap incorporation. Then, $0.3 \mathrm{mM}$ GTP was added into the reaction mixture and incubated at $37^{\circ} \mathrm{C}$ for 1 hour. Then, $1 \mu$ of TURBO DNase was added into the reaction mixture to remove the remaining template DNA and incubated at $37^{\circ} \mathrm{C}$ for another 15 minutes. After the incubation was complete, $30 \mu \mathrm{l}$ nuclease-free water and $30 \mu \mathrm{l}$ Lithium Chloride $(\mathrm{LiCl})$ precipitation solution was added to stop the reaction. The mixture was chilled for 30 minutes at $-20^{\circ} \mathrm{C}$ and centrifuged at $14,000 \mathrm{rpm}$ at $4^{\circ} \mathrm{C}$ for 15 minutes to pellet the RNA which then resuspended in nuclease-free water.

\subsection{Mechanical inoculation of tobacco plants with chimeric PVX viral transcripts}

Two types of inocula i.e. capped viral transcripts and plant sap virions were applied in Nicotiana tabacum. The capped viral transcripts of empty PVX and recombinant PVX::VP2 vector in the amount of 0.1$0.5 \mu \mathrm{g}$ were mechanically inoculated together with silicon carbide (400 mesh) on two fully expanded leaves by gently rubbing the leave surface. The viral sap inoculum of empty PVX and recombinant PVX::VP2 were prepared by homogenizing $0.5 \mathrm{~g}$ of previously infected Nicotiana tabacum leaves harvested at 4 days post infection (dpi) in $2 \mathrm{ml}$ of $0.1 \mathrm{M}$ phosphate buffer ( $\mathrm{pH} 7.0$ ). The homogenized leaves were centrifuged for 5 minutes at $12,000 \mathrm{~g}$ at $4^{\circ} \mathrm{C}$ to remove cell debris. For sap-inoculation of tobacco plants, the sap inoculum was diluted at 1:2 (v/v) in $20 \mathrm{mM}$ phosphate buffer. Two leaves per plant were inoculated with 10-20 $\mu 1$ of diluted inoculum per leaf. The inoculated plants were maintained in a growth room at $25^{\circ} \mathrm{C}$ with supplementary of lighting to give a 12 hour photoperiod.

\subsection{Extraction of plant total soluble protein and analysis of the expressed VP2 protein}

The total soluble protein was extracted from either fresh or frozen leaves of inoculated and noninoculated tobacco plants. Initially, leaf samples were ground into powder form in liquid nitrogen. Then, 0.5 $\mathrm{g} / \mathrm{ml}$ protein extraction buffer containing $50 \mathrm{mM}$ Tris- $\mathrm{HCl}, 150 \mathrm{mM} \mathrm{NaCl}, 0.2 \%$ Triton $\mathrm{X}-100$ and $1 \mathrm{mM}$ phenylmethylsulfonyl fluoride (PMSF) were added. The soluble protein was precipitated in 4 volumes of $10 \%$ TCA/aceton, chilled at $-20^{\circ} \mathrm{C}$ for 1 hour and centrifuged at $15,000 \mathrm{~g}$ for $10 \mathrm{~min}$ at $4^{\circ} \mathrm{C}$. The pellet was washed in cold acetone and air-dried before resuspended in rehydration buffer. The extracted proteins were separated by sodium dodecyl sulfate polyacrylamide gel electrophoresis (SDS-PAGE) before transferred onto a transfer membrane in Western blotting. The expressed VP2 protein was detected by primary antibody of polyclonal antiIBDV (Abcam, USA) and secondary antibody of polyclonal goat anti-chicken conjugated with horseradish peroxidase (HRP) (Abcam, USA). Western blot analysis was conducted according to the standard protocol described previously [27]. 


\subsection{Immunization of VP2 protein in rats and SPF chickens}

The immunogenicity of plant-based VP2 protein was evaluated by administrating the plant total soluble protein infected with empty PVX and PVX::VP2 in Albino Wistar rats. Approximately $150 \mu \mathrm{g}$ of total soluble protein was mixed with equal volume of complete Freund's adjuvant (Sigma, USA) for the first booster and incomplete Freund's adjuvant (Sigma, USA) for the second booster. The plant protein was administered via the intraperitoneal route. Blood was withdrawn via tail vein and sera were collected at day 0 and after the first (day 10) and second (day 20) inoculation of the protein. The sera were tested for the presence of anti-IBDV by dot blot assay. The immunogenicity of plant based VP2 protein in animal model was further evaluated by administration of the total soluble protein in specific-pathogen-free (SPF) chickens of 3 weeks old via subcutaneous route. This assessment was conducted as a pilot study in order to identify the immunogenicity level of the expressed VP2 protein. Approximately, $150 \mu \mathrm{g}$ of total soluble proteins was mixed with equal volume of complete Freund's adjuvant (Sigma, USA) in the first booster and incomplete Freund's adjuvant (Sigma, USA) was mixed with the protein in the second booster which administered two weeks after the first administration. About $0.5 \mathrm{ml}$ of blood was withdrawn via jugular vein and sera were collected before the inoculation (day 0) and after the second booster (day 28). The collected sera were tested for the presence of antiVP2 by enzyme-linked immunosorbent assay (ELISA) assay.

\subsection{Analysis of immunized serum by dot blot assay}

The immunized rat's serum collected at 0,10 and 20 days post inoculation (dpi) were analyzed for the presence of anti-VP2 neutralizing antibodies. In this analysis, the whole viral antigen of hvIBDV was spotted onto the polyvinylidene difluoride (PVDF) membrane. Then, the membrane was incubated in blocking solution containing 5\% skimmed milk in phosphate buffer saline (PBS) for 1 hour to block non-specific site of the membrane. After blocking, the membrane was incubated for 1 hour with the rat's serum as primary antibody collected at day 0,10 and 20 dpi previously immunized with the total protein of plants infected with empty PVX and PVX::VP2. Each serum sample was diluted at ratio 1:500 in 1X PBS and directed against the spotted IBDV antigen. After incubation, the membrane was washed three times in 1X PBS containing 0.1\% Tween20 (PBS-T) by incubating the membrane for 5 minutes in every wash. Then, the membrane was incubated for 1 hour with secondary antibody of goat polyclonal anti rat IgG conjugated with horseradish peroxidise (HRP) (Abcam, USA) diluted at 1:10000 in PBS-T. After incubation, the membrane was washed three times in PBS-T and once with 1X PBS. The membrane was incubated in DAB substrate (Pierce, USA) for few minutes until the desired signal was developed. The substrate reaction was stopped by washing the blot in distilled water.

\subsection{Analysis of antibody titer by enzyme-linked immunosorbent assay (ELISA)}

The enzyme immunoassay was conducted using IBDV antibody test kit from IDEXX (USA) according to the protocol recommended by the manufacturer. The test samples were diluted five hundred fold (1:500) with sample diluent and $100 \mu \mathrm{l}$ of diluted samples of each treatment were dispensed into triplicate wells. The plate was incubated for 30 minutes at $26^{\circ} \mathrm{C}$. The liquid content in each well was aspirated and washed 3 times with approximately $350 \mu \mathrm{l}$ of distilled water. After that, $100 \mu \mathrm{l}$ of Conjugate was dispensed into each well followed by 30 minutes incubation at $26^{\circ} \mathrm{C}$. Then, the liquid content was removed and washed with distilled water as described above. After washing steps, $100 \mu \mathrm{l}$ of TMB substrate was dispensed into each well and incubated for 15 minutes at $26^{\circ} \mathrm{C}$. Finally, $100 \mu \mathrm{l}$ of Stop Solution was dispensed into each well to stop the reaction. Then, the signals developed from each well were measured by a microplate reader at absorbance value of $650 \mathrm{~nm}$.

\section{RESULTS \\ 3.1 Amplification and cloning of hvIBDV gene in $P V X$ vector}

Analysis by $1 \%$ agarose gel electrophoresis of the PCR amplified VP2 gene from cDNA showed single bands of the expected size of $1.4 \mathrm{~kb}$ as indicated in Fig. 2. PCR screening and restriction enzyme (RE) analyses of the recombinant PVX vectors had detected the presence of VP2 gene. PCR screening of the PVX::VP2 recombinant vector showed single band of approximately $1.4 \mathrm{~kb}$ from all the 5 samples as shown in Fig. 3. For RE analysis, the recombinant PVX::VP2 vector produced two separated bands, i.e. $10 \mathrm{~kb}$ derived from the original PVX vector and $1.4 \mathrm{~kb}$ DNA of the VP2 fragment after digestion with EcoRV and SalI enzymes as shown in Fig. 4. 


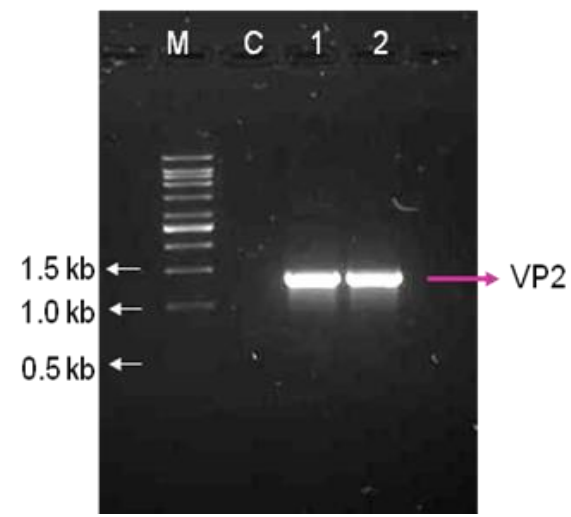

Fig. 2. PCR profile of hvIBDV VP2 gene ( 1.4 kb) amplified from cDNA samples (Lane 1 and 2), no template control (Lane C) and $1 \mathrm{~kb}$ DNA ladder (Vivantis, USA) (Lane M).

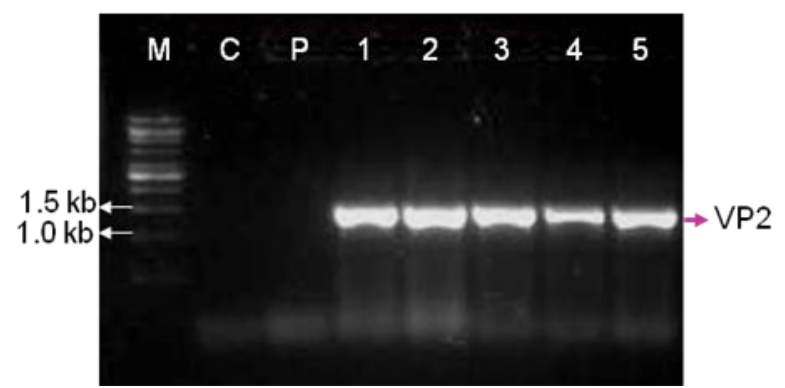

Fig. 3. PCR screening profile of hvIBDV VP2 gene $(\sim 1.4 \mathrm{~kb})$ from the recombinant PVX::VP2 vectors (Lane 1 to 5), PVX without VP2 insert (Lane P), no template control (Lane C) and $1 \mathrm{~kb}$ DNA ladder (Vivantis, USA) (Lane M).

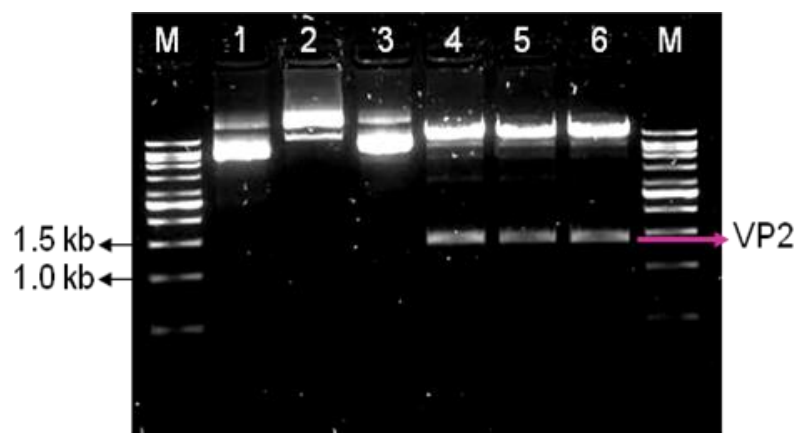

Fig. 4. Restriction profiles of the recombinant PVX::VP2 vectors using EcoRV and Sall enzymes. Undigested PVX vector without insert (Lane 1), digested PVX vector without insert (Lane 2), undigested recombinant PVX::VP2 vector (Lane 3), digested recombinant PVX::VP2 vector (Lane 4 to 6), and $1 \mathrm{~kb}$ DNA ladder (Vivantis, USA) (Lane M).

\subsection{Western blot analysis of VP2 expressed protein}

The presence of expressed VP2 protein in plants infected with PVX::VP2 was assessed by Western blot analysis. Total soluble proteins (TSP) were harvested from Nicotiana tabacum plants inoculated with either capped viral transcripts or plant sap virions. A prominent band of approximately $50 \mathrm{kD}$ was detected from protein sample inoculated with the capped viral transcripts (Fig. 5). A single band of approximately $80 \mathrm{kDa}$ was detected from protein sample inoculated with the plant sap virions (Fig. 6). These results demonstrated that the VP2 protein can be expressed either as a $50 \mathrm{kDa}$ protein or as a fusion epitope with the PVX coat protein (80 $\mathrm{kDa})$. 


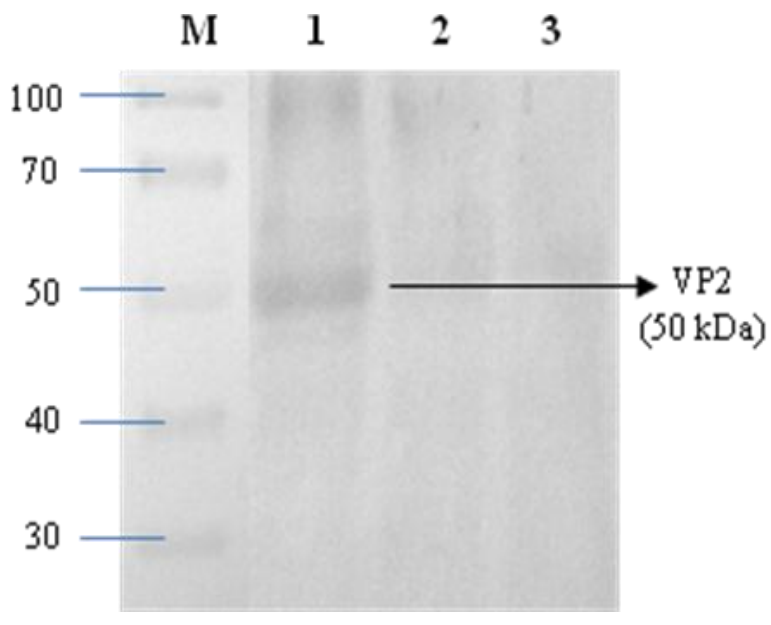

Fig. 5. Western blot analysis of total soluble protein of non-inculated leaf (Lane 3) and inoculated leaves infected with capped viral transcripts of empty PVX (Lane 2) and PVX::VP2 (Lane 1) harvested at 4 dpi. The size of expressed protein was estimated by Spectra Multicolour Broad Range Protein Ladder (Lane M).

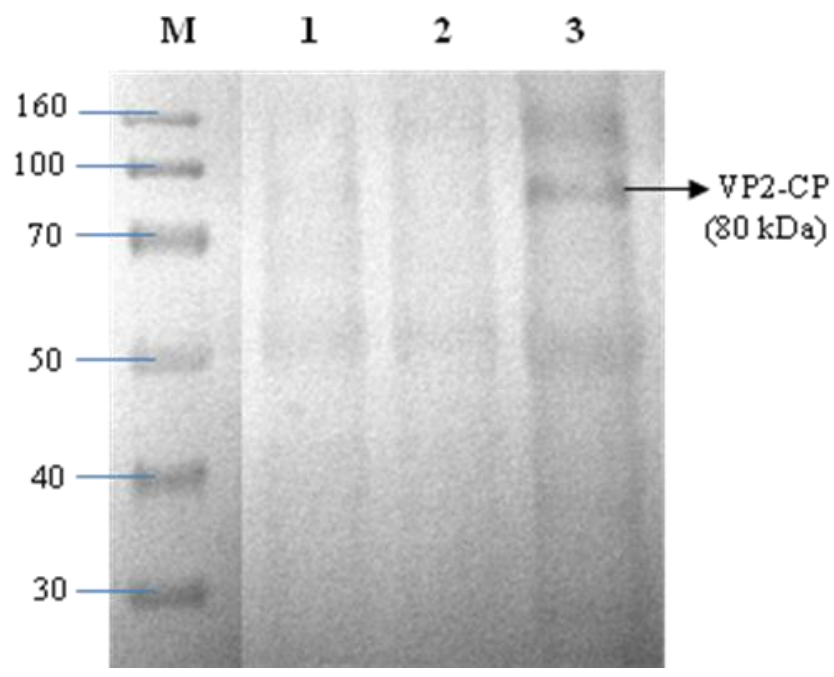

Fig. 6. Western blot analysis of total soluble protein of non-inculated leaf (Lane 1) and inoculated leaves infected with plant sap inoculum of empty PVX (Lane 2) and PVX::VP2 (Lane 3) at ratio 1:2 harvested at $4 \mathrm{dpi}$. The size of expressed protein was estimated by Spectra Multicolour Broad Range Protein Ladder (Lane M).

\subsection{Dot blot analysis of immunized rat serum}

Dot blot analysis of the serum derived from immunization of rats with plant total soluble protein containing the recombinant PVX::VP2 collected at day 10 showed signal when immunoreacted against the hvIBDV antigen and further increased after immunoreacted with serum collected at day 20 as demonstrated in Fig.7. On the other hand, there was no signal detected from the hvIBDV antigens immunoreacted with rat's serum immunized with total protein derived from plants infected with empty PVX as shown in Fig. 8. 


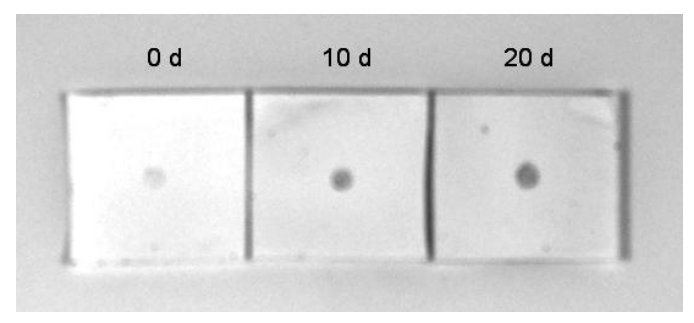

Fig. 7. Dot blot analysis of hvIBDV whole viral antigen immunoreacted with serum collected from rats immunized with plant total soluble protein containing the recombinant PVX::VP2

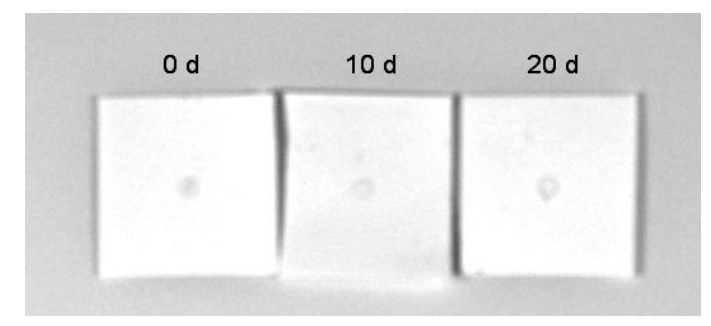

Fig. 8. Dot blot analysis of hvIBDV whole viral antigen immunoreacted with serum collected from rats immunized with plant total soluble protein containing the empty PVX vector

\subsection{Enzyme-linked immunosorbent assay (ELISA) of chicken serum}

The presence of neutralizing antibodies against the IBDV antigen in sera collected from the SPF chickens immunized with plant total soluble protein were analyzed by ELISA. Analysis of the sera previously immunized with the plant total protein containing the PVX::VP2 indicated detection of antibody titer (849) which exceeds the standard value of 391 as shown in Table 1. High antibody titer was detected from the serum collected at 28 days post-inoculation indicated the presence of immunogenic VP2 protein in the total soluble protein from Nicotiana tabacum inoculated with PVX::VP2 viral transcripts.

Table 1. Analysis of antibody titer from serum samples of immunized SPF chickens detected by ELISA

\begin{tabular}{|c|l|c|c|c|c|}
\hline \multirow{2}{*}{ No } & \multirow{2}{*}{ Samples } & \multicolumn{2}{|c|}{ 0 day (Before inoculation) } & \multicolumn{2}{c|}{ 28 days (After second inoculation) } \\
\cline { 3 - 6 } & & Titer & Antibody status & Titer & Antibody status \\
\hline 1. & Control (-ve) & 144 & Negative & 242 & Negative \\
\hline 2. & Empty PVX & 240 & Negative & 280 & Negative \\
\hline 3. & PVX::VP2 & 61 & Negative & 849 & Positive \\
\hline
\end{tabular}

Titer range: 390 or less $=$ Negative; 391 and above $=$ Positive

\section{DISCUSSION}

The present study was aimed at developing an alternative subunit vaccine candidate for the prevention of highly virulent infectious bursal disease (hvIBD). In this study, a full length of VP2 gene isolated from local strain of hvIBDV was identified and used for the expression of VP2 protein using plant viral-based transient expression system in tobacco plants. Previously, the proteins of chicken anemia virus (CAV) were cloned into a PVX viral vector [29]. The recombinant viral vector was transformed into Agrobacterium tumefaciens and delivered into the Nicotiana benthamina plants. The VP2 protein from virulent strain of IBDV has been expressed via plant viral-based epitope presentation strategy [24]. The truncated VP2 protein (24.5 kDa) was expressed as chimeric viral particles (CVPs) fused to the N-terminus of the Bamboo mosaic virus (BaMV) coat protein $(\mathrm{CP})$. In the present study, the whole VP2 protein structure $(50 \mathrm{kDa})$ was expressed using Potato virus $\mathrm{X}$ (PVX) transient expression vector. The VP2 gene of hvIBDV was cloned into a modified PVX vector, namely $\mathrm{pP} 2 \mathrm{C} 2 \mathrm{~S}$ and expressed under the control of duplicated subgenomic promoter of PVX CP.

Previous studies have reported the ability of pP2C2S vector to deliver various gene inserts including jellyfish green fluorescent protein (GFP) and the $\beta$-glucuronidase (GUS) gene [26] [28]. In the present study, an attempt has been made by incorporating the pP2C2S vector with the VP2 gene of approximately $1.4 \mathrm{~kb}$ in size in between the triple gene block and the coat protein. In comparison with the previous studies, the size of VP2 gene cloned onto pP2C2S vector was two times larger than the GFP and GUS gene. Restriction enzyme analysis 
revealed that the VP2 gene was successfully cloned onto the pP2C2S vector. The expression of VP2 protein in Nicotiana tabacum revealed detection of two versions of expressed VP2 protein based on the format of viral expression vector. Expression of VP2 protein using plant sap virus revealed detection of $80 \mathrm{kDa}$ protein band whereas the expression using capped viral transcripts revealed detection of VP2 protein as a $50 \mathrm{kDa}$ protein. Based on these findings, it can be predicted that the VP2 protein $(\sim 50 \mathrm{kDa})$ can be expressed as a fusion epitope together with the PVX CP $(\sim 30 \mathrm{kDa})$ when inoculated with plant sap virus. The expression of VP2 protein as a fused product with the PVX CP can be speculated due to mutation of the duplicated subgenomic promoter on the PVX viral vector that initiates the translation of the cloned gene. Since the expression of VP2 protein in pP2C2S employed the duplicated subgenomic promoter to drive exogenous gene expression [26], it can be speculated that mutation in the duplicated subgenomic promoter might contribute to the expression of VP2 protein as a fused translational product with the coat protein of PVX. A similar finding was reported previously [30] whereby the neutralizing epitope from human immunodeficiency virus type 1 (HIV-1) was expressed as Nterminal translational fusion with the PVX CP in Nicotiana benthamiana.

The immunogenicity assessments of plant based vaccine for IBDV have been described previously [23] [31]. Chicken orally fed with transgenic plants containing the expressed VP2 protein produced antibody against IBDV when analyzed by Western blot [23]. Specific-pathogen-free (SPF) chickens orally vaccinated with transgenic rice seeds produced neutralizing antibodies against IBDV and were protected when challenged with a highly virulent IBDV strain, BC6/85 [31]. In the present study, the immunogenicity of plant based VP2 protein expressed in tobacco plants was assessed by employing rats and chickens as animal models. Analysis of the serum collected from rats inoculated with the VP2 protein in plant crude extract revealed detection of signal when immunoreacted against the hvIBDV viral antigen. Chicken immunized with plant total soluble protein containing the expressed VP2 protein showed detection of antibody titer at day 28 post-immunization. These findings indicated that the VP2 protein expressed in Nicotiana tabacum was immunogenic and capable to induce immune response in animal models.

\section{CONCLUSION}

The present study had proven the feasibility of VP2 gene, approximately $1.4 \mathrm{~kb}$, from the Malaysian strain of hvIBDV to be cloned and expressed via PVX virus based transient expression system in the Nicotiana tabacum plants. The VP2 protein was successfully expressed either as an exogenous protein or a fusion protein with PVX CP. The expressed VP2 protein was found immunogenic and capable to induce immune response when immunized in animal models. By conducting this study, an alternative plant-based vaccine based on the complete structure of hvIBD VP2 protein from a Malaysian strain could be developed.

\section{Acknowledgements}

The authors would like to extend sincere appreciation to Prof. Dr. David Baulcombe (University of Cambridge, UK) for kindly supplying the Potato Virus $\mathrm{X}$-based expression vector, $\mathrm{pP} 2 \mathrm{C} 2 \mathrm{~S}$. This study is financially supported by the Ministry of Science, Technology and Innovation (MOSTI), Malaysia.

\section{REFERENCES}

[1] H. N. Lasher, and S. M. Shane, Infectious bursal disease, World's Poultry Science Journal, 50, 1994, $133-166$.

[2] H. Becht, and H. Muller, Infectious bursal disease-B cell dependent immunodeficiency syndrome in chickens. Behring Inst. Mitteilungen, 89, 1991, 217-225.

[3] M. M. Nagarajan, and F.S.B. Kibenge, Infectious bursal disease virus: A review of molecular basis for variations in antigenicity and virulence. Can. J. Vet. Res., 61(2), 1997, 81-88.

[4] J. Rong, X. N. Liu, and T. P. Cheng, Application of reverse transcription polymerase chain reaction (RT-PCR) for diagnosis of wild IBDV infection of chicks after to be inoculated IBDV vaccine. Chin. J. Vet. Sci. Technol. 31, 2001, 18-19.

[5] J. Giambrone, and J. Closser, Efficacy of live vaccines against serologic subtypes of infectious bursal disease. Avian Diseases, 34, 1990, 7-11.

[6] J. C. Muskett, N. E. Reed, D. H. Thornton, Increased virulence of an infectious bursal disease live virus vaccine after passage in chicks. Vaccine, 3, 1985, 309-312.

[7] K. J. Fahey, K. Erny, and J. A. Crooks, conformational immunogen on VP2 of infectious bursal disease virus that induces virusneutralizing antibodies that passively protect chickens. J. Gen. Virol., 70, 1989, 1473-1481.

[8] S. K. Reddy, A. Silim, and M. J. Ratcliffe, Biological roles of the major capsid proteins and relationships between two existing serotypes of infectious bursal disease virus. Arch. Virol., 127, 1992, 209-222.

[9] A. R. Omar, C. L. Kim, M. Hair-Bejo, and I. Aini, Efficacy of VP2 protein expressed in E.coli for protection against highly virulent infectious bursal disease virus. J. Vet. Sci., 7, 2006, 241-247.

[10] C. D. Bayliss, R.W. Peters, J. K. Cook, R. L. Reece, K. Howes, M. M. Binns, and M.E. Boursnell, A recombinant fowlpox virus that expresses the VP2 antigen of infectious bursal disease virus induces protection against mortality caused by the virus. Arch Virol., 120, 1991, 193-205.

[11] I. Shaw, and T.F. Davison, Protection from IBDV-induced bursal damage by a recombinant fowlpox vaccine, fpIBD1, is dependent on the titre of challenge virus and chicken genotype. Vaccine, 18, 2000, 3230-3241.

[12] M. Sheppard, W. Werner, E. Tsatas, R. Mccoy, S. Prowse, and M. Johnson, Fowl adenovirus recombinant expressing VP2 of infectious bursal disease virus induces protective immunity against bursal disease. Arch Virol., 143, 1998, 915-930. 
[13] R. Darteil, M. Bublot, E. Laplace, J. F. Bouquet, J. C. Audonnet, and M. Riviere, Herpesvirus of turkey recombinant viruses expressing infectious bursal disease virus (IBDV) VP2 immunogen induce protection against an IBDV virulent challenge in chickens. Virology, 211, 1995, 481-490.

[14] K. Tsukamoto, C. Kojima, Y. Komori, N. Tanimura, M. Mase, and S. Yamaguchi, Protection of chickens against very virulent infectious bursal disease virus (IBDV) and Marek's disease virus (MDV) with a recombinant MDV expressing IBDV VP2. Virology, 257, 1999, 352-362.

[15] V. N. Vakharia, D.B. Snyder, J. He, G.H. Edwards, P. K. Savage, and S. A. Mengel-Whereat, Infectious bursal disease virus structural proteins expressed in a baculovirus recombinant confer protection in chickens. J. Gen. Virol. 74, $1993,1201-1206$.

[16] Y. Liu, Y. Wei, X. Wu, and Y. Lian, Preparation of ChIL-2 and IBDV VP2 fusion protein by baculovirus expression system. Cell \& Mol. Immun., 2, 2005, 231- 235.

[17] C.W. Pei, Y.S. Hung, H.L. Long, T.L. Der, C.Y. Pei, and J.L. Hung, Secreted expression of the VP2 protein of very virulent infectious bursal disease virus in the methylotrophic yeast Pichia pastoris. J. Virol. Meth., 123, 2005, 221-225.

[18] D. M. Floss, and D. Falkenburg, Production of vaccines and therapeutic antibodies for veterinary applications in transgenic plants: an overview. Transgenic Research, 16, 2007, 315-332.

[19] J. K. Ma, P. M. Drake, and P. Christou, The production of recombinant pharmaceutical proteins in plants. Nat. Rev. Genet., 4, 2003, 794-805.

[20] E. Stoger, M. Sack, L. Nicholson, R. Fischer, and P. Christou, Recent progress in plantibody technology. Curr. Pharm. Des. 11, $2005,2439-2457$.

[21] M. M. Rigano, and A. M. Walmsley, Plant production systems and developments in plant-made vaccines. Immunology and Cellular Biol. 83(3), 2005, 271-277.

[22] A.M. Walmsley, and C.J. Arntzen, Plants for delivery of edible vaccine. Current Opinion in Biotechnology, 2, 2000, 126-129.

[23] H. Wu, N. K. Singh, R.D. Locy, K. Scissum-Gunn, and J. J. Giambrone, Expression of immunogenic VP2 protein of infectious bursal disease virus in Arabidopsis thaliana. Biotechnol Lett. 26, 2004, 787-792.

[24] T. H. Chen, T. H. Chen, C. C. Hu, J. T. Liao, C. W. Lee, J. W. Liao, M. Y. Lin, H. J. Liu, M. Y. Wang, N. S. Lin and Y. H. Hsu, Induction of protective immunity in chickens immunized with plant-made chimeric Bamboo mosaic virus particles expressing very virulent Infectious bursal disease virus antigen. Virus Research, 166, 2012, 109-115.

[25] P. Chomczynski, and N. Sacchi, Single-step method of RNA isolation by acid guanidinium thiocyanate-phenol-chloroform extraction. Anal. Biochem. 162, 1987, 156-159.

[26] D. C. Baulcombe, S. Chapman, and S. Santa Cruz, Jellyfish green fluorescent protein as a reporter for virus infections. Plant J., 7, 1995, 1045-1053.

[27] H. Towbin, Origins of protein blotting. In B. T. Kurien, \& R. H. Scofield (Eds.), Methods in molecular biology, protein blotting and detection. Humana Press: Totowa, New Jersey, 536, 2009, 1-3.

[28] S. Chapman, T. Kavanagh, and D. Baulcombe, Potato virus X as a vector for gene expression in plants. Plant J., 2, $1992,549-557$.

[29] C. Lacorte, H. Lohuis, R. Goldbach, and M. Prins, Assessing the expression of chicken anemia virus proteins in plants. Virus Research, 129, 2007, 80-86.

[30] C. Marusic, P. Rizza, L. Lattanzi, , C. Mancini, M. Spada, F. Belardelli, E. Benvenuto, and I. Capone, Chimeric plant virus particles as immunogens for inducing murine and human immune responses against human immunodeficiency virus type $1 . J$. Virol., 75, 2001, 8434-8439.

[31] J. Wu, L. Yu, L. Li, J. Hu, J. Zhou, and X. Zhou, Oral immunization with transgenic rice seeds expressing VP2 protein of infectious bursal disease virus induces protective immune response in chickens Biotechnology J., 5, 2007, 570-578. 